\title{
Grado de importancia de las finanzas y costos en el sector empresarial
}

Rafael Roosell Paez Advincula ${ }^{1}$

\begin{abstract}
RESUMEN
El objetivo de esta investigación es mostrar el análisis financiero y de costos integrados que ha cobrado importancia en el mundo empresarial, evaluar el desempeño financiero, los costos operativos; detectar dificultades y aplicar correctivos para la toma de decisiones de la empresa. Se ha realizado un estudio mediante una revisión exhaustiva que ha permitido desarrollar los temas de costos y finanzas en el sector empresarial, el cual ha ido ganando mayor importancia en el análisis y modelos de toma de decisiones de inversión en el ámbito empresarial, con la finalidad de que los empresarios tengan la capacidad de identificar la viabilidad de sus respectivas inversiones para mejorar la rentabilidad.
\end{abstract}

Palabras-claves: Liquidez; rentabilidad; viabilidad; beneficio económico.

DEGREE OF IMPORTANCE OF FINANCE AND COSTS IN THE BUSINESS SECTOR

\section{ABSTRACT}

The objectives of this research are to show the integrated financial and costs analysis that has gained importance in the business world, to evaluate financial performance and operative costs, to correct problems, and to apply corrective measures for company decision-making. A study carried out through an exhaustive review made it possible to expand upon cost and finance matters in the business sector, which has gained greater importance in investment analysis and decision-making models in the business sector, so that business leaders are able to identify the viability of their respective investments to improve profitability.

Keywords: Liquidity; profitability; viability; economic performance.

\section{INTRODUCCIÓN}

La presente investigación documental pretende mostrar cómo ha cobrado importancia el estudio de los costos dentro del campo de las finanzas en general, para ello se utiliza métodos históricos para describir la evolución de los aspectos verdaderamente resaltantes de este estudio, al igual que se realiza una investigación desde el punto de vista descriptivo con el fin de dar a conocer los fenómenos que han logrado el avance cada vez más creciente de los costos en el ámbito de las finanzas. La meta de una empresa es ganar dinero, pero dentro de ciertas restricciones, ante ello surge la pregunta: ¿serán rentables las mencionadas inversiones? Se ha realizado múltiples estudios en el planeta, y aparentemente, la inclinación es conveniente, aunque aún no es algo categórico. Antiguamente el valor se fijaba por medio de la suma de costos y un margen de beneficio o herramienta definido por las propias compañías, en la actualidad, el precio generalmente se encuentra definido por el mercado.

¿Qué tiene que ver las finanzas y costos con el sector empresarial?, según parece, el ámbito de las finanzas y el tema de los costos son asuntos divergentes. No obstante, existe en la actualidad en el ámbito empresarial una inclinación realmente creciente de personas e instituciones que orienta sus reglas estratégicas de inversión en la toma de decisiones, valoración de los activos, construcción de carteras, gestión de costos y finanzas corporativas. Podríamos hacernos la interrogante sobre si esta incorporación de criterios de finanzas y costos es realmente ventajosa.

Ante la conjetura: ¿Las empresas son más rentables cuando desarrollan un modelo estratégico de finanzas y costos cuando se encuentran vinculadas?, se puede pensar que el uso realmente eficiente de los recursos financieros presenta en sí mismo un valor de costo empresarial. De tal modo, la rentabilidad de la empresa significa, en un inicio, que los elementos que se le han confiado fueron aplicados con eficacia y han rendido frutos. Además es posible pensar que existen costos en beneficio de la empresa, lo cual es muy probable, y sería aún mejor que sea regido

1 Corporación Industrial Paez S.A.C.

Ingeniero Industrial de la Universidad Nacional Mayor de San Marcos. Lima, Perú

E-mail: operaciones@incorpaezsac.com

Código ORCID: htps://orcid.org/000-0003-1413-7246 
mediante una regularización motivada en el análisis y la elección del modelo de toma de decisiones en el sector empresarial.

La operación de la empresa supone realizar una sucesión de ocupaciones o funcionalidades que en términos en general se tiene la posibilidad de congregar las cinco categorías: Producción, Marketing, Recursos humanos, Costos y Finanzas, las cuales se encuentran anexadas de una forma similar e independiente para la maximización del valor de la empresa. Las decisiones de inversión tienen una propiedad que las distingue principalmente en la toma de decisiones financieras: ellas forman parte de todas las superficies funcionales de la empresa.

\section{METODOLOGÍA}

Arias (2012) "La investigación descriptiva consiste en la identificación de un hecho, fenómeno, individuo o grupo, con el objetivo de establecer su estructura o comportamiento" (p.24), plantea que la investigación descriptiva implica por parte del investigador la capacidad de exponer, en forma minuciosa los estudios que ponga en manifiesto los conocimientos desde el punto de vista teórico y metodológico. Es por ello, que el presente estudio pretende realizar un análisis financiero y de costos en relación con la toma de decisiones mediante una investigación aplicada.

El tipo de investigación fue descriptivo porque es descrito el comportamiento de las variables de estudios y el diseño sobre el que se basa los estudios administrativos que permiten construir elementos que ayudan a identificar las características fue no experimental.

Durante el proceso de investigación se utilizó la fuente de información de los estados financieros de la empresa, también se realizó a partir de textos, documentos, artículos, páginas web, entre otros.

Brealey (2015) afirma : "El secreto del éxito en la administración financiera es incrementar el valor" (p.2), el establecimiento de un análisis financiero que en un pequeño o largo plazo constituirá una ventaja competitiva, y al mismo tiempo será un medio para tratar de optimizar sus ingresos y mejorar ampliamente sus márgenes de utilidad, criterios científicos, técnicos, entre otros; para lograr emitir una opinión oportuna sobre las condiciones financieras de la empresa, se procura medir el desempeño dentro del contexto del negocio en el cual se lleva a cabo los propósitos y tácticas.
Myers (2016) afirma: "Un plan financiero coherente demanda la comprensión de la forma en que la empresa puede generar rendimientos superiores en largo plazo" (p.804), intenta definir el análisis detallado y permite determinar cuáles son los factores clave de un negocio, lo que se refleja como requisito indispensable para una proyección razonable de la circunstancia económica y financiera futura de la empresa. Un plan financiero se basa sobre la toma de la información de uno o varios de los estados financieros de un periodo y de esta forma se logra analizar las relaciones que hay entre ellos $y$ el negocio en desarrollo, define las finanzas empresariales y al papel que juegan las finanzas en el alcance del éxito empresarial, así mismo define quién es el encargado del manejo financiero dentro de una empresa, cuáles son las funciones del administrador financiero, qué relación existe entre las finanzas y costos del conjunto de actividades que realizan en una empresa o como inciden el entorno empresarial o en el desarrollo de las funciones del administrador financiero.

Lawrence (2003) detalla: "El estado de resultado proporciona un resumen financiero de los resultados de las operaciones de la empresa durante un periodo determinado"(p.53), define un informe financiero que fundamentalmente detalla la rentabilidad que se genera en una empresa durante un periodo determinado; es decir, las ganancias y pérdidas de la empresa, ello facilita contrastar el desempeño de la empresa en todo el tiempo y/o contrastar el desempeño de la compañía en relación a la industria de la que forma parte, los estados financieros y económicos a usar son:

Estado de resultados.- es un reporte que muestra y detalla ingresos y gastos que se utilizan en un periodo determinado con el objetivo de analizar y tomar decisiones.

Balance general.- por definición es un estado que sintetiza la posición financiera, tiene como misión realizar una comparación entre los activos, pasivos y el patrimonio en cuanto a su economía y financiamiento de las cuentas por cobrar o deuda de capital que aportaron los socios durante la formación de la empresa.

Flujo efectivo.- tanto el efectivo como los valores negociables representan principalmente una reserva de liquidez que en la mayoría de los casos aumenta o disminuye los efectivos.

Flujo operativo.- Se define las entradas y salidas de efectivo que van directamente con la producción de productos y servicios. 
Flujo de inversión.- se encuentra vinculado con la adquisición y venta tanto de activos fijos como de inversión de negocio.

\section{RESULTADO DEL RENDIMIENTO ECONÓMICO}

"El análisis financiero es un instrumento fundamental disponible para la gerencia y sirve para predecir el efecto que pueden generar algunas decisiones estratégicas en el desempeño futuro de la empresa; decisiones como la venta de una dependencia, variaciones en las políticas de crédito, en las políticas de cobro o de inventario, así como también una expansión de la empresa a otras zonas geográficas" (Brigham y Houston,2006,p.831), define como una utilidad clave para el manejo del desempeño económico y financiero de la empresa.

Rengifo y Court (2001) afirma : "Estos modelos son de bastante uso en las finanzas, usualmente las predicciones obtenidas con estos modelos son mejores que las obtenidas con los modelos estructurales" (p.339), intenta saber el resultado financiero, rentabilidad, márgenes de herramienta por la línea de producto, medir la eficacia en la utilización correcta de los elementos, detectar donde es posible comenzar a reducir costos, establecer un precio de referencia (cotizaciones), y también consigue valorizar los inventarios.

\section{Medir los costos transcendentales}

\section{a. Costo por área}

Costo de producción.- materias primas, insumos, mano de obra

Costo de administración.- alquiler, suministro de oficina
Costo de comercialización.- publicidad, marketing

Costo de financiamiento.- amortización e intereses de créditos

\section{b. Costo por variabilidad}

Costos fijos: se mantienen constantes e independientes del nivel de actividad de la empresa (alquileres, gasto administrativo)

Costo variable: aumento o disminución de manera proporcional según el nivel de producción de la empresa (materia prima, mano de obra directa)

Van Horne (2010) afirma:" La mayoría de las decisiones financieras, tanto personales como de negocios consideran el valor dinero en tiempo" (p.42), define la gestión de tiempo y espacio debe dar lugar de importancia en la agenda de cualquier empresa al hecho de lograr niveles saludables de servicio al cliente, rentabilidad operativa, económica y financiera.

\section{Análisis de valor de producción}

Se puede ver en la figura 1 , que el valor minuto a tener en cuenta en el sector de operaciones en un rango o fluctuación de valor minuto de: <0.190-0.212] soles/min; para que los costos fijos de la planta sean absorbidos.

\section{c. Según su relación}

Costo directo: se establece directamente al volumen de producción, se asimila a los costos variables.

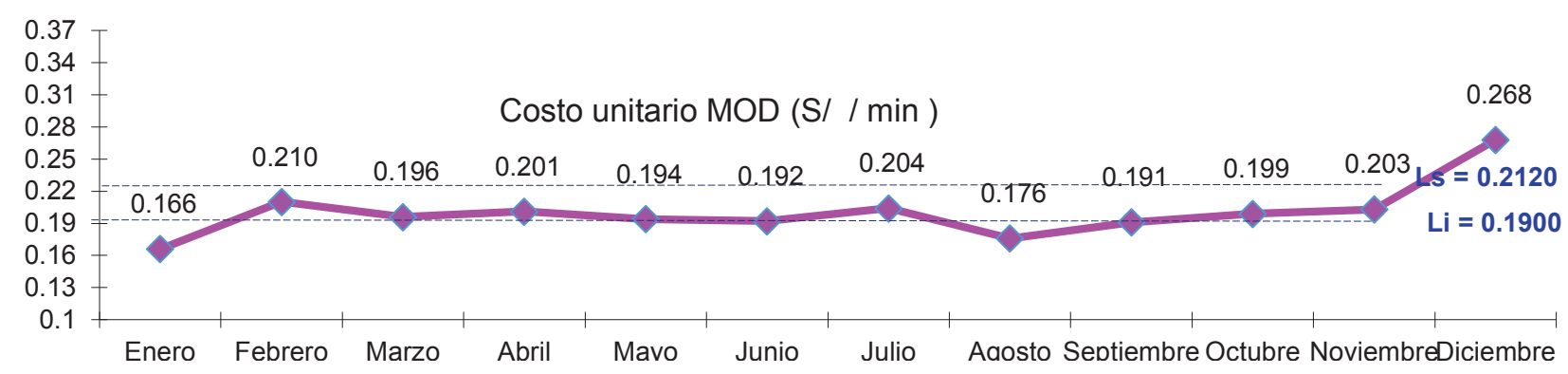

Figura 1. Costo valor minuto

Fuente: Elaboración propia 
Costos indirectos: no se establecen de manera directa a un producto o servicio, sino que se distribuyen entre las distintas entidades productivas y se asimilan a los costos fijos.

Welch (2005) afirma:" El plan táctico de utilidades debe estructurarse en forma tal que satisfaga las necesidades de construcción de los costos de los productos, costos de los proyectos y costos de otra naturaleza" (p.91), define la categorización de los costos como dependiente en concordancia a su variabilidad y se clasifican por su función, tiempo, comportamiento y relación, como aparecen en la tabla 1.

Amat y Soldevila (2011) afirma:" Menciona que los costos se clasifican según la finalidad que la organización necesite" (p.26), define a los costos como portadores de un papel muy sustancial en el desarrollo de toma de decisiones de la empresa.

Costo variable.- Se establece en concordancia al nivel de actividad, varía de manera directa según el volumen, se utiliza algunas oportunidades en forma proporcional con las ventas o el volumen de producción.

Se observa en la figura 2, que el costo variable total se incrementa de manera directa con la producción y se duplica la producción, el costo variable se duplica o si la producción disminuye en un $5 \%$, el costo disminuye en un $5 \%$, en cambio, el costo de la variable unitario sigue recurrente.
Costo fijos.- se establece con las estructuras o capacidad instalada de la empresa, que esos costos que están en el transcurso de un tiempo determinado no se encuentran concordantes con el volumen de producción.

Se puede apreciar en la figura 3, que el costo fijo total se mantiene constante con la producción, en cambio, el costo fijo unitario disminuye en un $50 \%$ si se duplica la producción, por lo tanto, el costo fijo unitario se encuentra en una relación indirectamente proporcional con referencia a la producción.

Rosas (2006) afirma: "Grandes transacciones económicas y capitales nacionales e internacionales brindan un panorama amplio y diversificado de lo que pasa cuando las fuerzas económicas impactan" (p.599), define una visión del mundo desde una perspectiva financiera, para hacer un proyectado o estimación, se emplea el lado derecho del balance para las empresas que trabajan activamente en el mercado de capitales.

Lawrence (2003) afirma:" El balance general presenta un estado resumido de la situación financiera de la empresa en un momento especifico" (p.56), define una clase de foto que retrata la circunstancia contable de la compañía en un periodo de tiempo, el inversionista tiene una información vital de cómo se encuentra el negocio o cuál es la disponibilidad de liquidez, solvencia y rentabilidad.

Tabla 1. Estructuración de los costos de acuerdo a su variabilidad

\begin{tabular}{|c|c|c|c|}
\hline Área & Tiempo & Comportamiento & Relación \\
\hline Producción & \multirow[t]{2}{*}{ Histórico } & Variable & \multirow{2}{*}{ Directos } \\
\hline Administración & & Fijo & \\
\hline Marketing y comercial & \multirow[t]{2}{*}{ Presupuesto proyectado ( estimados, estándares y normales) } & & \multirow{2}{*}{ Indirectos } \\
\hline Finanzas & & Semifijo o semivariable & \\
\hline
\end{tabular}

Fuente: Elaboración propia

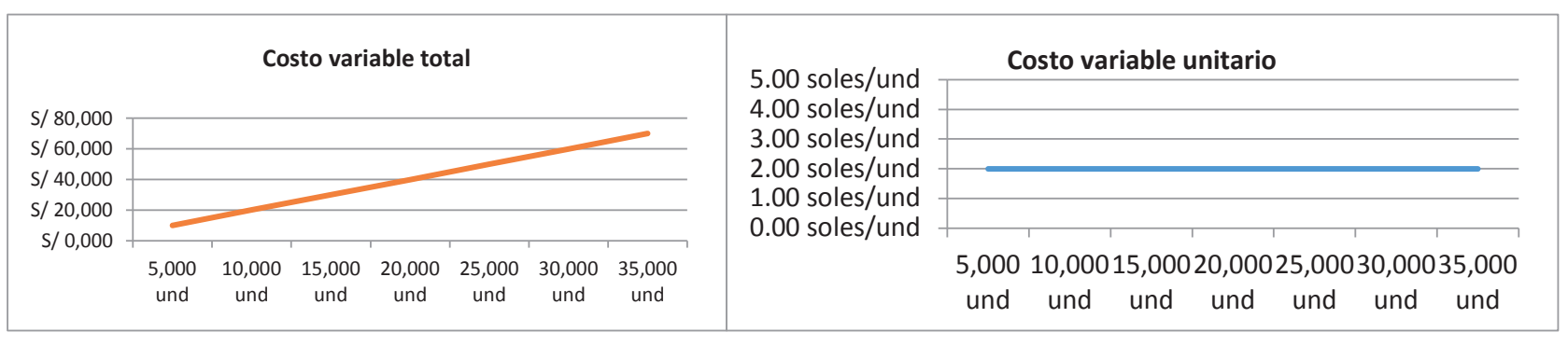

Figura 2. Costo variable

Fuente: Elaboración propia 


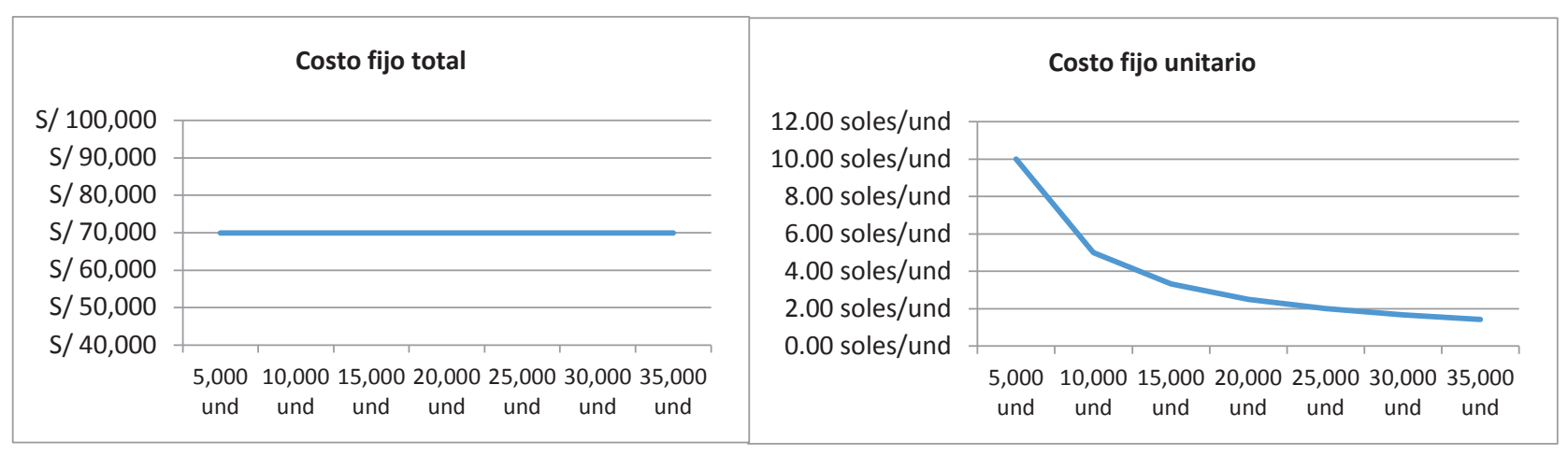

Figura 3. Costo fijo

Fuente: Elaboración propia

Tabla 2. Balance general

\begin{tabular}{|l|l|}
\hline Activo & Pasivo y Patrimonio \\
\hline \multirow{4}{*}{$\begin{array}{l}\text { Inversiones } \\
\text { Globales }\end{array}$} & Pasivo \\
\cline { 2 - 2 } & Facilidades bancarias \\
\cline { 2 - 2 } & Créditos de proveedores \\
\cline { 2 - 2 } & Pagarés bancarios \\
\cline { 2 - 2 } & Emisión de bonos \\
\cline { 2 - 2 } & Acciones \\
\cline { 2 - 2 } & Patrimonio \\
\cline { 2 - 2 } & Capital Social \\
\cline { 2 - 2 } & Reserva de Capital \\
\cline { 2 - 2 } & Utilidades acumuladas \\
\hline
\end{tabular}

Fuente: Elaboración propia

Se observa en la tabla 2, las partidas tienen sus propios valores para cada periodo, se logra deducir la proporción o el porcentaje del financiamiento total de cada fuente (pasivo o patrimonio) en el financiamiento total y se logra también efectuar el análisis, la planeación financiera y de costo para la toma de decisiones.

\section{Pautas para obtener un reporte que apoye al pronóstico de la empresa:}

- La innovación de la información financiera a una manera más útil para supervisar y predecir la condición financiera de la compañía.

- Evaluar la necesidad de aumentar o disminuir la aptitud productiva.

- Determinar el financiamiento requerido.

Establecer decisiones financieras.- Es requisito planificar el financiamiento a corto y largo plazo en un tiempo preciso, por otro lado es necesario saber cuáles son las fuentes particulares superiores del mismo desempeño puesto que la escasez de normas dicta las pretensiones, diagnósticos económicos, financieros y los costos operativos que se atribuyen. Muchas de estas pretensiones necesitan un diagnóstico más profundo de las decisiones financieras y sus costos operativos.

Herramienta para el administrador financiero.- El responsable financiero consigue tomar decisiones con el objetivo de lograr los objetivos de liquidez y rentabilidad estipulados por la empresa, necesita las siguientes utilidades financieras:

- Análisis de indicadores financieros de liquidez, solvencia y endeudamiento que permiten conocer las fortalezas y debilidades financieras para tomar decisiones preventivas y correctivas.

- Los presupuestos determinan por anticipado las proyecciones de liquidez, solvencia y rentabilidad de la empresa, nos permite evaluar y controlar la gestión administrativa.

- Las matemáticas financieras nos facilitan considerar proyectos de inversión, calcular el valor del capital, valor del desarrollo operativo, calcular el desempeño efectivo, saber maneras de amortizar deudas, tomar decisiones de adquisición o arrendamiento financiero.

- Técnicas de administración de activos y pasivos corrientes, influyen en los criterios que guían el manejo de liquidez, solvencia y rentabilidad.

- Estado de fuentes y uso de fondos, establecer y considerar la calidad de las decisiones 
gerenciales de los recursos de la empresa con la forma de cómo fueron comprados.

- La contabilidad de costo utiliza conceptos como el costo fijo, variable, punto de equilibrio, margen de contribución y de utilidad.

La optimización de las utilidades a largo plazo de la corporación, se debe considerar normas y política de actividades que desarrollan el proceso operativo y financiero de la empresa, dado que esta tiene una relación directa de la misma manera que el ámbito generalmente.

\section{COSTOS Y FINANZAS CLAVES PARA EL ÉXITO EMPRESARIAL}

Términos como finanzas, costos, flujo de caja, estado financiero, presupuesto, entre otros, abarcan mucho más de lo que se lee: comprenderlo es fundamental en el ámbito de los negocios.

Lawrence (2003) afirma: "La mayoría de las decisiones empresariales se miden en términos financieros, el gerente financiero desempeña un papel clave en la operación de la compañía"(p.7), la administración financiera efectivamente comprende análisis, reportes, diagnósticos del periodo operativo de la empresa, el valor del dinero para el negocio, relaciones con los inversionistas, la contabilidad, el estudio y la comunicación, acertadamente un extenso rango de stakeholders, lo cual implica los emprendimiento; gestión de activos, presupuesto, pronósticos, gestión de los costos y el fluido de efectivo.
Cuando se habla de criterios como finanzas y costos, hay que comprender que son utilidades indispensables para el avance y desarrollo de alguna compañía, además con sus aportes se consigue una adecuada interpretación resultados y se puede tomar decisiones rápidas y guiar las acciones hacia un óptimo desempeño. La experiencia indica que los mencionados criterios se encuentran dentro de las primordiales superficies de optimización en la administración, ya que la mayor parte trabaja más por intuición que por idealización.

Finanzas y costos, brazo derecho de todo negocio.- Es el registro, clasificación de todos los documentos organizados y clasificados por fechas, tiene como función estudiar, medir e investigar el patrimonio de las organizaciones para la toma de decisiones y el control de la información se muestra de forma sistemática para las diferentes partes con el objetivo de maximizar la rentabilidad dentro de la organización.

Westerfield (2005) afirma:" Las corporaciones deben presentar un cálculo de la utilidad o perdida tanto a sus propios accionistas como a las autoridades" ( $p .179)$ plantea maximizar y optimizar los recursos en las áreas operativas dentro de la organización para un desarrollo sostenible del valor del dinero.

Myers (2010) afirma. "Las corporaciones eligen una meta de estructura de capital que maximiza el valor de la empresa "(p.523) plantea maximizar el propósito primordial del responsable financiero que se encarga de maximizar el valor invertido de los inversionistas, tal cual se puede apreciar en la figura 4.

\section{OPTIMIZAR EL VALOR DE LA EMPRESA}

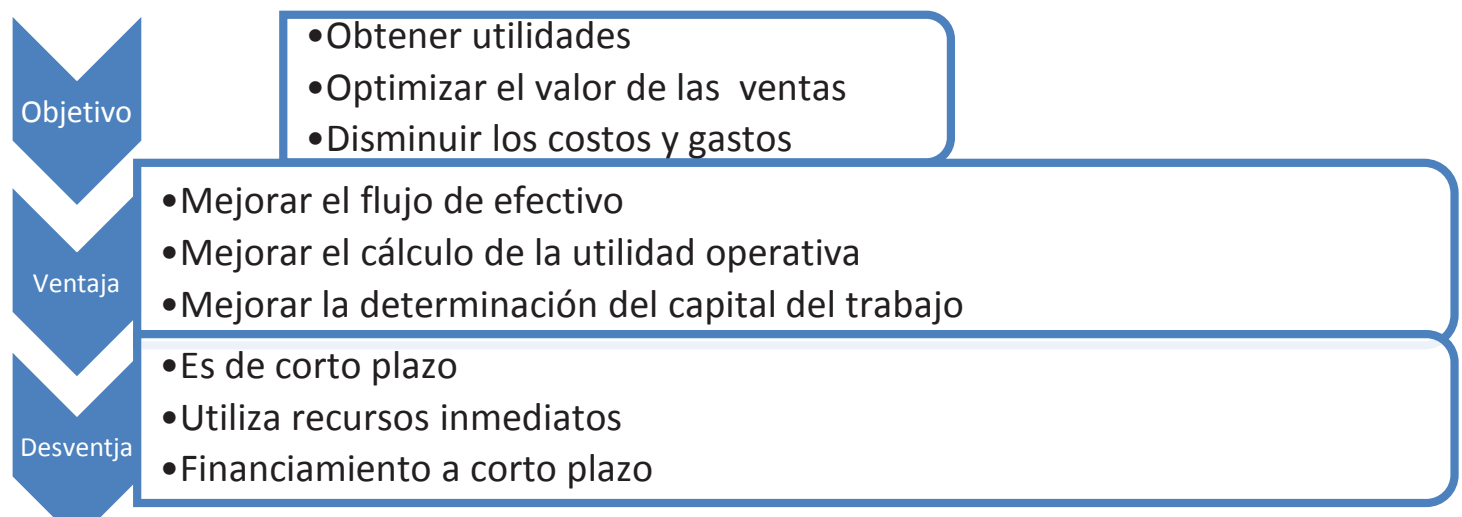

Figura 4. Maximizar el valor de la empresa Fuente: Elaboración propia 


\section{Informe financiero y costo integrado, tendencia mundial}

Los empresarios utilizan reportes de la situación económica y financiera de la empresa, de esta manera detecta los cambios que experimenta la misma. La información que brindan los estados financieros resulta ventajosa para la gestión de la empresa para ser más competitiva.

- Cada vez son más las empresas que tienen en cuenta su reporte semanal, mensual, semestral y anual de costos y finanzas con el valor sostenible.

- Expresan resultado de rentabilidad, liquidez y solvencia en un periodo determinado.

- Es un reporte orientado a tomar decisiones empresariales.

- Contribuye a medir la administración de la organización.

- Aporta información que ofrece diferentes superficies.

- Brinda seguridad a entes bancarios que podrían financiar algún emprendimiento de la empresa.

\section{Presupuesto, una herramienta fundamental.}

Westerfield (2005) afirma: "Qué métodos de presupuesto de capital deberían usar las empresas"(p.157) plantea el uso del plan financiero para administración de ingresos y gastos; es decir, tomar en cuenta la cantidad de dinero que se requiere y la cantidad que la empresa dispone para invertir o para financiar lo primordial y llevar a cabo el plan de negocio, para la empresa es menester tener esta utilidad ya que ayuda en el desarrollo de la estrategia como se puede apreciar en la figura 5 .

\section{Presupuesto de inversión.-}

Nos permite establecer elementos requeridos para el avance de un emprendimiento. En la formulación de este plan intervienen el aspecto legal, fiscal, am- biental, etc. Por esta razón hay que tener en cuenta la normativa vigente de acuerdo con la zona donde se va a realizar el emprendimiento.

\section{Control del flujo de efectivo}

Esta herramienta nos permite tener información sobre las variaciones de entradas y salidas de efectivo. Es la acumulación neta de activo líquido en un determinado lapso y constituye la liquidez de la empresa.

La administración financiera orienta las decisiones que la empresa toma respecto de sus flujos de efectivo, en consecuencia, la administración financiera es medular. El tipo de operaciones que abarca la gama de la administración financiera se muestra al tomar decisiones como ampliar la planta, automatizar las áreas productivas, adquirir nuevos activos, etc.

Meyers (2010) afirma:" Las empresas pueden pronosticar sus necesidades de efectivo y diseñar un plan financiero de corto plazo para enfrentar cualquier escasez de efectivo" (p.795). En consecuencia, ello permite ver en números el futuro de la empresa, para pronosticar o proyectar las ventas, gastos e inversiones en un periodo determinado, como se grafica en la figura 6 .

Los gerentes de finanzas también tienen el compromiso de decidir sobre los términos de crédito que van a poder prestar de las entidades financieras para que el proyecto continúe con un respaldo económico sostenible, para ello se debe realizar un análisis adecuado del volumen de inventario óptimo, de costos y gastos permitidos por la empresa para realizar un análisis de factibilidad de compra de recursos lo que finalmente permitirá generar mayores utilidades y liquidez a mediano y a largo plazo. Para ello se requiere:

- Informes sobre el estado de los ingresos y egresos de efectivo.

- Proporcionar información relevante para personas inversionistas y administradoras.

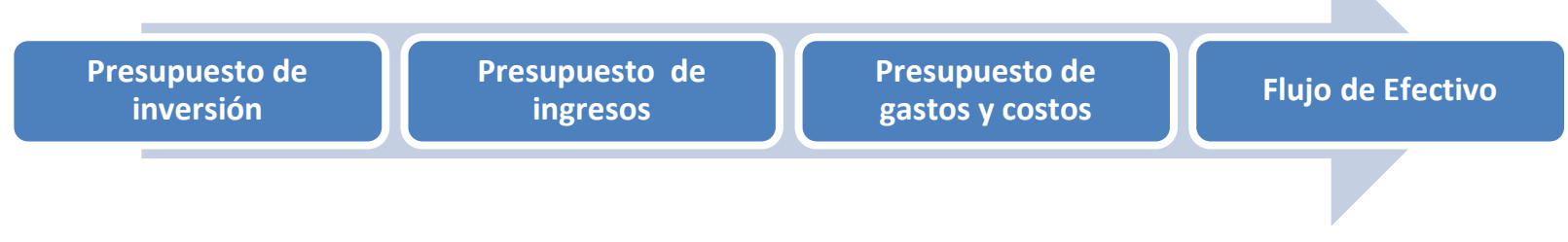

Figura 5. Tipos presupuestos

Fuente: Elaboración propia 


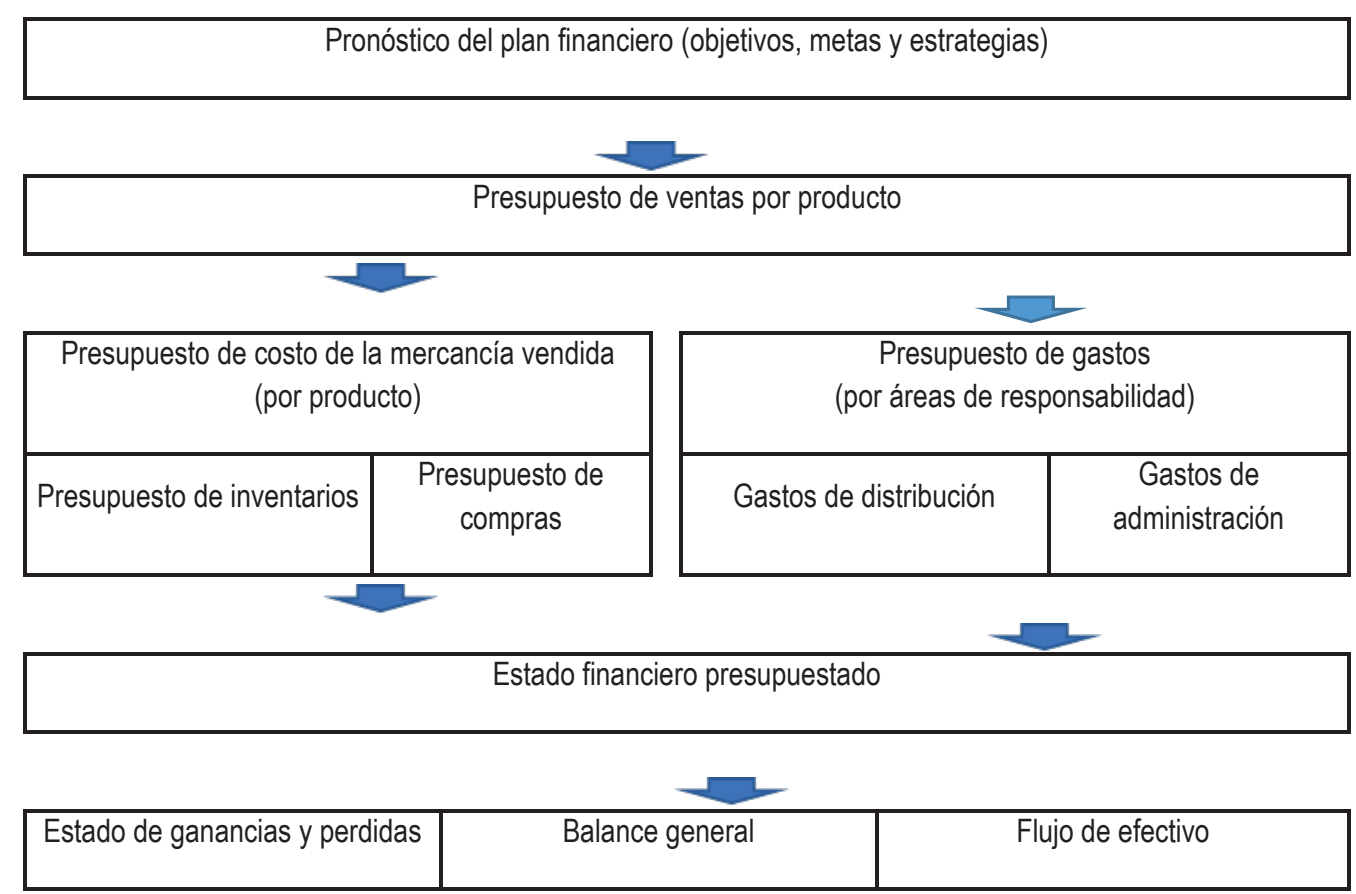

Figura 6. Pronóstico del plan financiero

Fuente: Elaboración propia

- Evaluar la aptitud de la compañía para crear el flujo de efectivo positivo.

- Determinar la aptitud del negocio para cumplir sus obligaciones.

- Facilitar la identificación de pretensiones de financiamiento.

- Identificar las partidas que más afectan a la empresa.

Hirache (2013) afirma: "El flujo de caja también conocido como 'cash flow' muestra el estado de los ingresos menos los egresos de efectivo de una empresa" planea el indicador financiero que permitirá encaminar la capacidad de generación de liquidez, ahí es donde se evidencia la importancia de las proyecciones financieras, se debe considerar el beneficio de la empresa ya que el beneficio contable va a llevar siempre el importe de las amortizaciones como las provisiones. A manera de ejemplo se presenta la tabla 3.

Se muestra primordialmente las actividades en los estados financieros con el básico propósito de calcular el flujo de efectivo para un respaldo económico de liquidez, solvencia y rentabilidad para el soporte económico del proyecto de la empresa al corto y largo plazo con un apalancamiento adecuado dentro de las entidades financieras.

\section{CONCLUSIONES}

Se puede concluir que ha ido tomando mayor consideración el análisis y los modelos para toma de decisiones de inversión en el ámbito empresarial, con el objetivo de que los inversionistas sean capaces de detectar la viabilidad de las inversiones para optimizar la rentabilidad de la cartera del inversor; la propuesta está respaldada por empresas que han invertido en este rubro. La investigación académica respecto a costos y finanzas, desde una perspectiva empresarial. Es fundamental para el empresario contar con técnicas de costos y finanzas que le beneficiarán en la viabilidad de cualquier proyecto en el sector económico empresarial.

El problema de liquidez, solvencia y rentabilidad se muestra únicamente en la toma de decisiones financieras a corto y largo plazo, en las cuales se detalla el volumen de adquisición de materiales, aplazamiento de pago a proveedores, la fluctuación y variación de los costos y gastos incurridos para el proyecto para no recurrir a un sobre giro bancario y contemplar un déficit de tesorería. El análisis financiero y de costos se considera importante para la gestión de la empresa, ya que esta solamente evalúa el aspecto interno, el incremento de la demanda interna y externa, su participación en el mercado nacional con las perspectivas o planes de creci- 


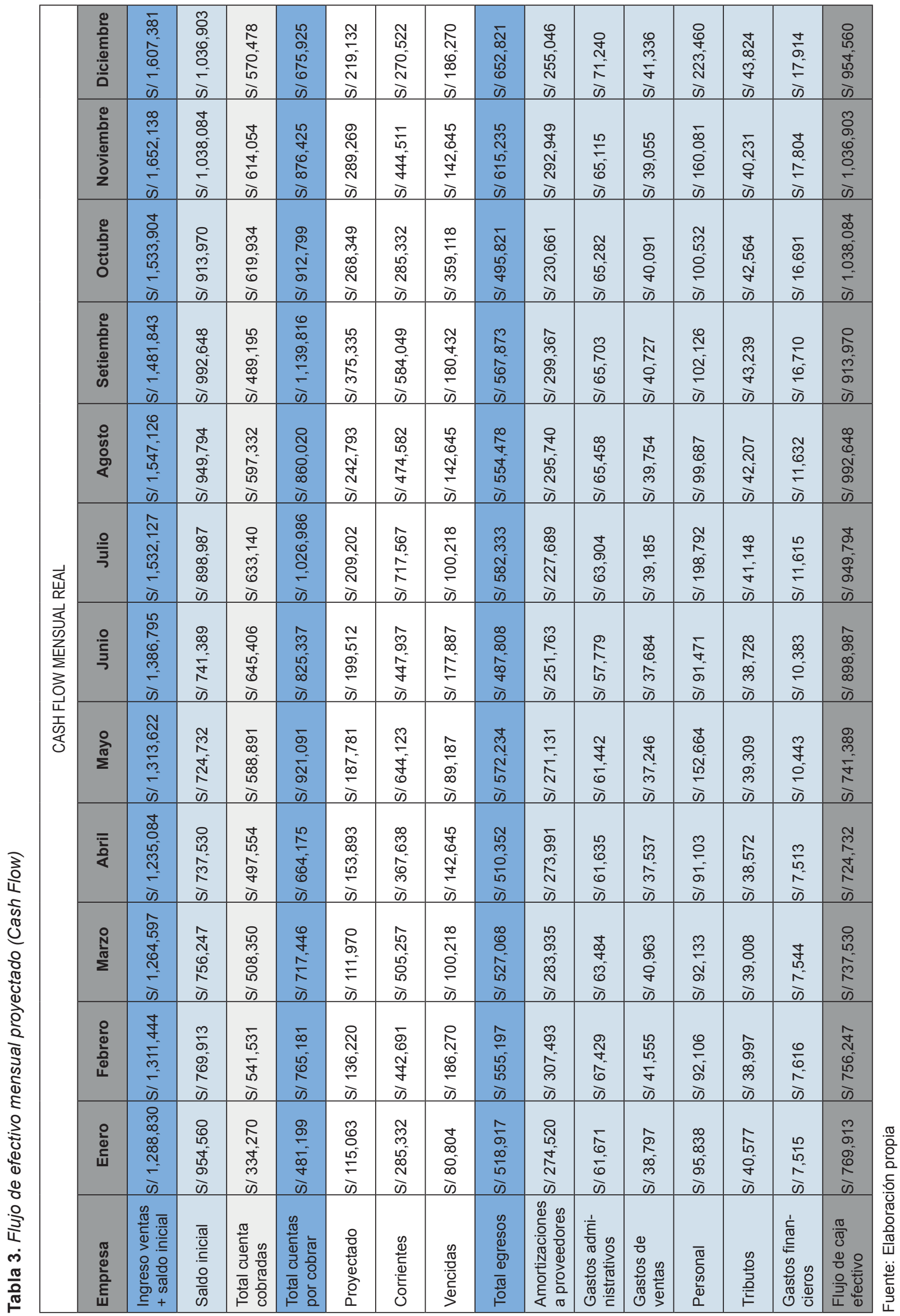


miento por los próximos años. La variación del precio unitario muestra el impacto de sensibilidad en la rentabilidad esperada, atribuyendo un escenario de viabilidad del proyecto de inversión de la empresa a un corto y largo plazo.

\section{RECOMENDACIONES}

Respecto al análisis financiero y de costo analizado podemos recomendar lo siguiente:

- Realizar análisis y modelos financieros para la toma de decisiones empresarial.

- Detectar la viabilidad de los costos del proyecto para los inversionistas.

- Utilizar técnicas y herramientas que nos permitan tomar decisiones oportunas.

- Reducir los costos de producción sin afectar la calidad para mejorar el precio y competir con productos extranjeros que hayan ingresado al mercado nacional.

- Conseguir financiamiento con las mejores tasas para mejorar la rentabilidad.

\section{REFERENCIA BIBLIOGRÁFICA}

[1] Amat, O. y Soldevilla, P. (2011). Contabilidad y gestión de costes (6ta ed.). España. Editorial: Profit.

[2] Arias, F.(2012). El proyecto de investigación (6ta ed.).Caracas, Venezuela. Editorial: Episteme

[3] Bae, K., Bailey, W., y Mao, C. (2006). Stock market liberalization and the information environment. Journal of International Money and Finance, 25, p. 404-428.

[4] Block, S. (2001). Fundamentos de gerencia financiera, (9 ed.), Bogotá, Colombia: Mc. Graw Hill.
[5] Brealey, R. (2015). Fundamentos de finanzas corporativas, (5 ed.), Madrid, España: Mc. Graw Hill.

[6] Brigham, E. y Houston, J. (2006). Fundamentos de Administración Financiera (10ª ed.), México: Cengage Learning Editores.

[7] Hirache, L. (2013). El flujo de caja como herramienta financiera. Revista actualidad empresarial, 277, p. 4-7.

[8] Lawrence, G. (2003). Principios de administración financiera, (10 ed.), México D.F. México: Prentice Hall.

[9] Mantilla, S. (2006). Información financiera IFRS (NIIF): Estándares normas internacionales. Bogotá, Colombia: ECOE.

[10] Myers, B. (2010). Principios de finanzas corporativas (9 ed.), Bogotá, Colombia: Mc. Graw Hill.

[11] Rengifo, E., y Court, E. (2011). Estadísticas y econometría financiera. Buenos Aires, Argentina: Cengage Learning.

[12] Rivera, j. y Alarcon D. (2012). El cargo de capital en la evaluación del desempeño financiero de empresa innovadoras de confecciones de cali, Estudios gerenciales, 28(123), p.87-92.

[13] Rosas, A. (2006). Antropología de la deuda: Crédito, ahorro, fiado y prestado en las finanzas cotidianas. Revista Mexicana de Sociología, 68, p. 599-602.

[14] Van Horne, J. y Wachowicz J. (2010) Fundamentos de administración financiera, (13 ed.), Mexico D.F. Mexico: Pearson

[15] Welch, G. (2005). Planificación y control, (6 ed.), México Editorial: Pearson

[16] Westerfield, R. (2005). Finanzas corporativas, (9 ed.), México Editorial: Mc. Graw Hill. 